\title{
The Development of Ternary Nanoplexes for Efficient Small Interfering RNA Delivery
}

\author{
Emine Şalva, ${ }^{*, a}$ Suna Özbaş Turan, ${ }^{b}$ and Jülide Akbuğa ${ }^{b}$ \\ ${ }^{a}$ Department of Pharmaceutical Biotechnology, Faculty of Pharmacy, Inonu University, Central Campuss; 44280, \\ Malatya, Turkey: and ${ }^{b}$ Department of Pharmaceutical Biotechnology, Faculty of Pharmacy, Marmara University; \\ Tibbiye Street, Istanbul 34668, Turkey. \\ Received June 4, 2013; accepted September 24, 2013; advance publication released online October 3, 2013
}

Targeted posttranscriptional gene silencing by RNA interference (RNAi) has garnered considerable interest as an attractive new class of drugs for several diseases, such as cancer. Chitosan and protamine are commonly used as a vehicle to deliver and protect small interfering RNA (siRNA), but the strong interaction still remains to be modulated for efficient siRNA uptake and silencing. Therefore, in this study, ternary nanoplexes containing chitosan and protamine were designed to substantially enhance the siRNA efficiency. Binary and ternary nanoplexes were prepared at different the ratios of moles of the amine groups of cationic polymers to those of the phosphate ones of siRNA (N/P) ratios and characterized in terms of size, zeta potential, morphology and serum stability. The silencing efficiencies and cytotoxicities of prepared nanoplexes were evaluated by enzyme-linked immunosorbent assay (ELISA) (for human vascular endothelial growth factor; hVEGF) and 3-(4,5-dimethylthiazol-2-yl)-2,5-diphenyltetrazolium bromide (MTT) assays, respectively. The mean diameter of ternary nanoplexes ranged from 151 to $282 \mathrm{~nm}$, depending on the weight ratio between polymers and siRNA. The gene silencing effect after transfection with ternary nanoplexes (chitosan/ siRNA/protamine $83 \%$ ) was significantly higher than that with binary nanoplexes (chitosan/siRNA $71 \%$ and protamine/siRNA 74\%). Ternary nanoplexes showed the highest cellular uptake ability when compared with binary nanoplexes. Ternary nanoplexes did not induce substantial cytotoxicity. Serum stability and the lack of cytotoxicity of the nanoplexes provided advantages over other gene silencing studies. These results suggest ternary nanoplexes have the potential to be an effective siRNA carrier to study the gene silencing effect.

Key words small interfering RNA; protamine; chitosan; ternary nanoplex; vascular endothelial growth factor

RNA interference (RNAi) is already a valuable and widelyused research tool in the analysis of molecular mechanisms for many diseases. In addition to being a research tool, RNAi holds great promise in terms of gene therapy to silence disease-causing genes. RNAi, which is mediated by small interfering RNA (siRNA), has been developed to suppress the expression of specific genes in mammalian cells. ${ }^{1)}$ Recently, this silencing mechanism has been widely used to knockdown cancer-associated genes, offering great promise in the field of cancer therapy. ${ }^{2)}$ Vascular endothelial growth factor (VEGF) and its receptors have been implicated in the angiogenesis that occurs in many solid tumours, including breast, colon, hepatoma, bladder, gastric and prostate cancers. Several strategies have been developed for targeting the VEGF pathway as a part of anticancer therapy. ${ }^{3)}$ RNAi-mediated silencing of oncogenic regulators that play major roles in tumour transformation, growth and metastasis is therefore considered a promising strategy for cancer therapy. ${ }^{4)}$

It is widely accepted that the problem of delivery is one of the major hurdles to turning siRNA into a therapeutic. This is because the hydrophilic and polyanionic structure of siRNA impede efficient delivery and uptake into cells. ${ }^{1)}$ Therefore, the therapeutic applications of siRNA have been focused on the development of stable and effective delivery systems. In the siRNA delivery, non viral vectors have been increasingly proposed as safer alternatives to viral vectors because they have many advantages such as being easy to produce in large quantities, remaining stable in storage, cell/tissue targeting, pos-

The authors declare no conflict of interest. sessing a low host immune response, their cell/tissue targeting ability and their capacity for unrestricted loading of genetic materials. ${ }^{5,6)}$ Among the non viral vectors, cationic polymers and cationic phospholipids have attracted a lot of attention, they interact electrostatically with negatively charged siRNA and then form complexes because of their cationic charge. ${ }^{5,7)}$

Numerous studies on DNA delivery with chitosan as a carrier biomaterial have shown effective expression of reporter and disease genes in vitro and in vivo, promoting chitosan as an attractive candidate for siRNA delivery. This is because it is a naturally derived, biocompatible, biodegredable and nontoxic biopolymer. ${ }^{8)}$ Chitosan nanoparticles and complexes have been shown to be effective for in vitro and in vivo delivery of siRNA. ${ }^{9,10)}$ Howard et al. observed a chitosan/siRNA nanoparticle-mediated knockdown of an enhanced green fluorescent protein in bronchiole epithelial cells. ${ }^{11)}$ Jere et al. suggested that chitosan-graft-polyethyleneimine (PEI) carrier efficiently and safely delivered siRNA. ${ }^{12}$

Recently, the ability of protamine to provide liposomes and nanoparticles which are useful for siRNA delivery has been tested. Protamine is a nontoxic, cationic and naturally occurring polypeptide which is known to provide unique membrane-translocating and nuclear localizing activities because of its arginine-rich sequence. ${ }^{13)}$ In addition to its stabilization enhancing properties, protamine is well known for transporting macromolecules into cells. It is known to exhibit cell penetrating activity and is an important component of several cancer targeting systems. ${ }^{14)}$ Song et al. used a protamine/antibody fusion protein to deliver siRNAs specifically to cells expressing the human immunodeficiency virus-1 
(HIV-1) envelope protein. ${ }^{15)}$ The positively charged protamine served as binding partner for negatively charged siRNA and showed cell internalization and release of the siRNA cargo. Choi et al. reported that a low molecular weight protamine could carry and localize siRNA inside tumors and inhibit the expression of VEGF through systemic application of peptide complex, thereby suppressing tumor growth. ${ }^{16)}$

Chitosan and protamine are commonly used as a vehicle to deliver and protect siRNA, but the strong interaction still remains to be modulated for efficient siRNA uptake and silencing. Therefore, ternary nanoplexes containing chitosan and protamine were designed to substantially enhance the siRNA efficiency. VEGF, which is frequently overexpressed in breast cancer, was used as a model system. Here we describe for the first time the use of chitosan/siRNA/protamine nanoplexes as a new delivery system for siRNA. In our study, this ternary nanoplexes were characterized, and their ability to down-regulate VEGF expression in different breast cancer cell lines and cell internalization is demonstrated.

\section{MATERIALS AND METHODS}

Materials Chitosan $(75 \mathrm{kDa}$ and $75-85 \%$ degree of deacetylation) and protamine sulfate salt $(5.1 \mathrm{kDa})$ were purchased from Sigma (St. Louis, MO, U.S.A.). Dulbecco's modified Eagle's medium (DMEM), fetal bovine serum (FBS), trypsinethylenediaminetetraacetic acid (EDTA) and penicillinstreptomycin were purchased from Gibco BRL (Rockville, MD, U.S.A.). siRNA-VEGF-A and mismatch siRNA-VEGF-A (Dharmacon, Lafayette, CO, U.S.A.) and ELISA kit for VEGF were used (Invitrogen, Camarillo, CA, U.S.A.). A thorough analysis by BLAST was performed on all siRNA sequences used in this study to demonstrate that these sequences have no homology to other genes. All the other chemicals were of cell culture and molecular biology grade.

Preparation of Ternary Nanoplexes Chitosan was dissolved in acetate buffer $(0.1 \mathrm{M}$ Tris acetate $/ 0.1 \mathrm{M}$ acetic acid, $\mathrm{pH} 5.4)$ to form of $0.25 \%(\mathrm{w} / \mathrm{v})$ chitosan solution. Protamine was dissolved phosphate buffered saline (PBS) to form a solution of $2.5 \mathrm{mg} / \mathrm{mL}(\mathrm{w} / \mathrm{v})$. Lyophilized siRNAs were dissolved in $5 \times$ siRNA buffer and final concentration was made $1 \mu \mathrm{g} /$ $\mu \mathrm{L}$. Chitosan/siRNA and protamine/siRNA binary nanoplexes (from $1 / 1$ to $20 / 1$; +/-) were prepared by adding chitosan and protamine solution to siRNAs stock solutions for forming of the nanoplexes completely. The ternary nanoplexes consisting of chitosan/siRNA/protamine were formed by adding of protamine to chitosan and siRNA mixing (chitosan/siRNA/ protamine nanoplexes, from $1 / 1 / 1$ to $20 / 1 / 20$; $+/-/+$ ). In the various N/P ratios (the ratios of moles of the amine groups of cationic polymers to those of the phosphate ones of siRNA) both protamine and chitosan for siRNA delivery were evaluated. To evaluate the condensation capability of binary and ternary nanoplexes, agarose gel electrophoresis $(2.5 \%, \mathrm{w} / \mathrm{v})$ were carried out under a voltage of $120 \mathrm{~V}$ for $30 \mathrm{~min}$. Images were obtained using a UV transilluminator and a digital imaging system (Kodak, U.S.A.).

Characterization of Nanoplexes Mean particle diameter (Z-average) and zeta potential (surface charge) of nanoplexes were determined using Malvern Zetasizer Nano ZS (Malvern, U.K.). The measurements were made at $25^{\circ} \mathrm{C}$ in triplicate. For both of the measurements, nanoplexes were suspended in PBS at $\mathrm{pH} 7.4$.

The morphological examination of nanoplexes were made by Scanning Electron Microscopy (SEM, Jeol, JSM 5200, Japan).

siRNA Stability Assay Protection of complexed siRNA against serum was studied by incubating the samples in $10 \%$ fetal bovine serum and $150 \mathrm{~mm} \mathrm{NaCl}$ solution at $37^{\circ} \mathrm{C}$. Chitosan/siRNA $(15 / 1 ;+/-)$ and chitosan/siRNA/protamine $(15 / 1 / 15 ;+/-/+)$ nanoplexes were prepared for serum stability studies. Reaction was carried out at $37^{\circ} \mathrm{C}$ and aliquots were taken at different intervals. For the inhibition of reaction $0.5 \mathrm{M}$ EDTA was used. Nanoplexes were then analyzed by agarose gel electrophoresis and the integrity of the siRNA in nanoplexes was compared to naked siRNA treated with serum.

In Vitro Transfection MCF-7 and MDA-MB435 breast cancer cell lines were from American Type Culture Collection (ATCC, Rockville, MD, U.S.A.) and used for transient transfection experiments. The cell lines were maintained in Dulbecco's minimum essential medium (DMEM) media supplemented with $10 \% \mathrm{FBS}$ and antibiotics at $37^{\circ} \mathrm{C}$ under $5 \% \mathrm{CO}_{2}$ incubator. Cells were plated in 24 -well plate at a cell density of $5 \times 10^{4}$ cells/well and incubated overnight. Transfections were performed on cells that were approximately $70 \%$ confluent. Protamine/siRNA and chitosan/siRNA binary nanoplexes and ternary nanoplexes (500 ng siVEGF for each well) were added to in 24-well plates and incubated for $48 \mathrm{~h}$ for transfection. The cells were washed twice with ice cold PBS and lysed in $100 \mu \mathrm{L}$ of lysis buffer by freezing and thawing (incubation at $-20^{\circ} \mathrm{C}$ for $30 \mathrm{~min}$ in a deep-freezer and then $37^{\circ} \mathrm{C}$ for $30 \mathrm{~min}$ ). Cell debris was removed by centrifugation at $12000 \times \boldsymbol{g}$ for $5 \mathrm{~min}$. The supernatant was transferred to a new microcentrifuge tube.

Cells were seeded on coverslips at densities of $5 \times 10^{4}$ cells/ well. Cells were transfected with nanoplexes containing fluorescein isothiocyanate (FITC)-labeled siVEGF. After $4 \mathrm{~h}$, cells were washed with PBS and fixed with $2 \%$ formaldehyde in PBS. Coverslips were mounted on glass slides and cells were analyzed with laser scanning spectral confocal microscope (Leica TCS SP2, Germany).

The Determination of VEGF Amount ELISA for VEGF was performed in the supernatant of the cell culture suspension, according to manufacturer's instructions (Biosource, CA, U.S.A). VEGF protein was analyzed using solid phase sandwich ELISA. Absorbance was determined by spectrophotometric reading at $450 \mathrm{~nm}$. All the experiments were repeated thrice and the standard deviation $( \pm)$ was calculated.

In Vitro Cytotoxicity To evaluate the cytotoxicity of chitosan and protamine nanoplexes, cell viability following nanoplexes treatment was measured with 3-(4,5-dimethylthiazol2-yl)-2,5-diphenyltetrazolium bromide (MTT) assay according to the manufacturer's instruction. MCF-7 and MDA-MB-435 cells were seeded into 96 well plates at the density of $5 \times 10^{3}$ cells/well and incubated at $37^{\circ} \mathrm{C}, 5 \% \mathrm{CO}_{2}$ for $24 \mathrm{~h}$ to allow cell attachment. Nanoplexes in the culture media were incubated with the cells for $48 \mathrm{~h}$ at $37^{\circ} \mathrm{C}$. After incubation with samples for $48 \mathrm{~h}$, culture medium was removed and replaced with fresh PBS containing $2 \mathrm{mg} / \mathrm{mL}$ MTT. The MTT-treated cell culture medium was further treated with dimethyl sulfoxide (DMSO) to dissolve the formazan crystals formed by live cells, then measured their absorbance at $570 \mathrm{~nm}$. Cell viability was expressed as percentage of absorbance in comparison with that 


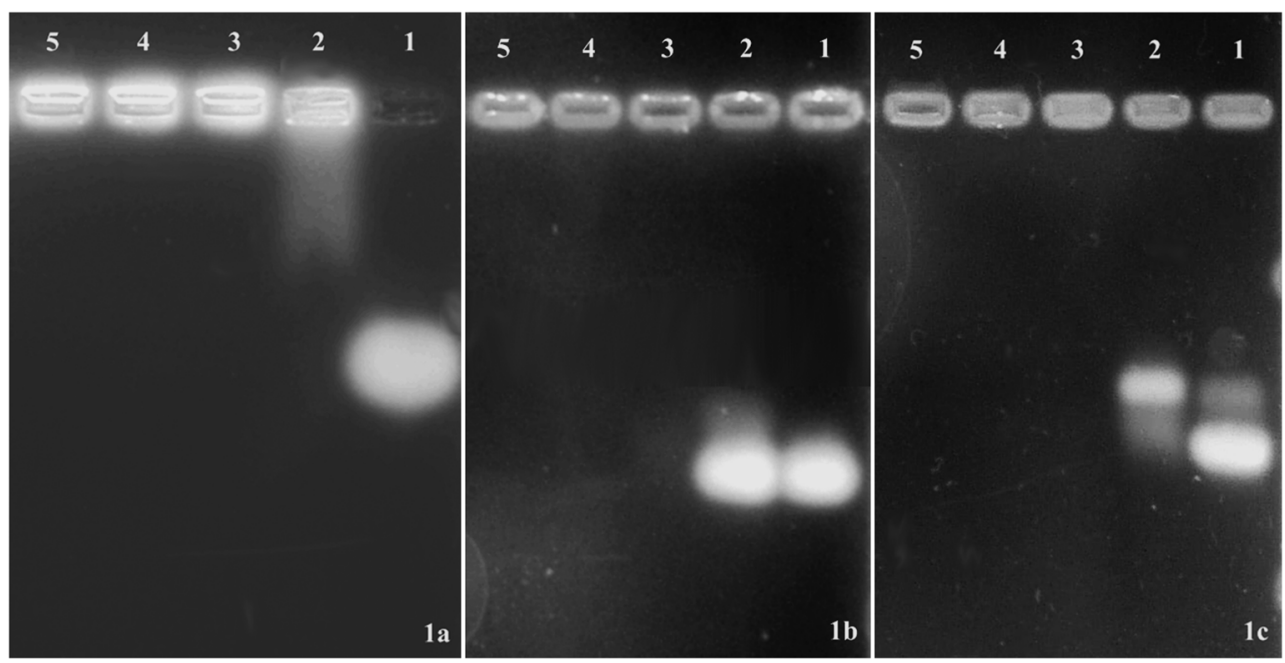

Fig. 1. Agarose Gel Electrophoresis of Nanoplexes at Various N/P Ratios

a. Chitosan/siRNA/protamine nanoplexes, b. Chitosan/siRNA, c. Protamine/siRNA (Lane 1: Free siVEGF, Lanes 2-5: 1/1, 5/1, 10/1, and 20/1, N/P).

of the control, which was comprised of cells treated with the culture medium.

Statistical Analysis Results are reported as means \pm standard deviation. Paired $t$-test was applied to assess the statistical difference among different groups.

\section{RESULTS AND DISCUSSION}

The new concept of protamine and chitosan based nanoplexes to deliver siRNA as a therapeutic agent directed against the VEGF gene shows interesting characteristics, with regard to average particle size and zeta potential, non-cytotoxicity, high stability and cellular uptake. In this study, chitosan/ siRNA/protamine nanoplexes as a new siRNA carrier were developed that are capable of delivering siRNA, through the cellular membrane and achieving high activity of RNAi.

Preparation and Control of Nanoplexes siRNA condensation ability is important for gene vectors, since it is neccessary to protect siRNA against nuclease degredation. The siRNA condensation behavior of polymer is attributed to the electrostatic interaction between polymer and siRNA molecules. ${ }^{17)}$ Gel retardation assay was carried out to evaluate the condensing ability of binary and ternary nanoplexes.

Different $\mathrm{N} / \mathrm{P}$ ratios of chitosan and protamine to siRNA were tested at fixed siRNA concentration (from $1 / 1$ to $20 / 1$ $(+/-)$ ratio) (Fig. 1). The complete retardation of the binary and ternary nanoplexes can be observed when the weight ratio of protamine and chitosan to siRNA was above $5 / 1(+/-)$ and $5 / 1 / 5(+/-/+)$, respectively. Below this ratios, partly complexation formed between polymer and siRNA. As shown in Fig. 1 , when the value of $+/-$ ratio of polymer/siRNA reached to $5 / 1$, free siRNA could not be detected on agarose gel. These results suggested that ternary nanoplexes were able to effectively condense siRNA into complex at the charge ratio $5 / 1 / 5$, as the migration of the formed nanoplex on agarose gel was considerably retarded due to charge neutralization and the increase in the overall molecular size. These findings are in good agreement with those observed by other investigators, indicating that protamine and chitosan formed a condensed complex with pDNA and siRNA as effectively as any of the currently used cationic polymeric gene carriers. ${ }^{18-20)}$
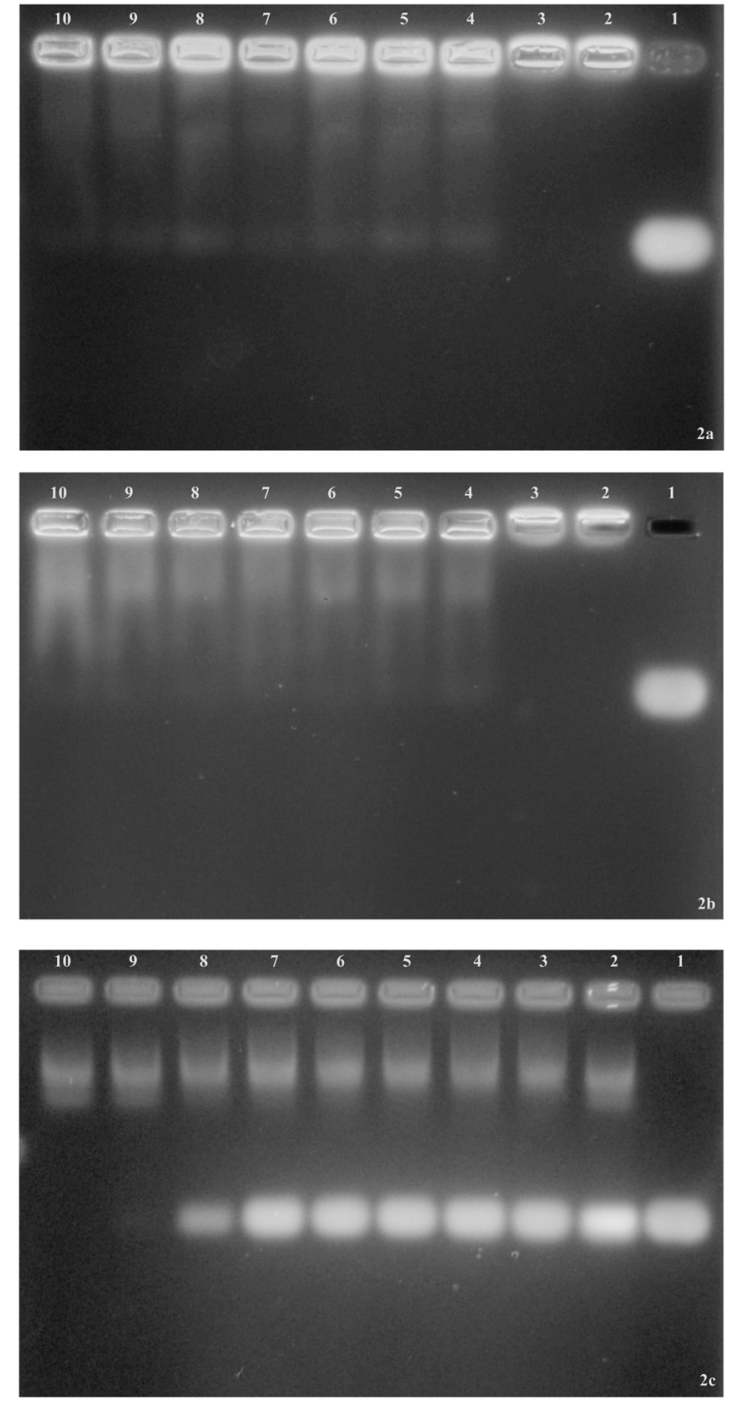

Fig. 2. Serum Stability of Chitosan/siRNA (15/1) and Chitosan/siRNA/ protamine (15/1/15) Nanoplexes

a. Ternary nanoplexes. Lane 1: Free siVEGF, Lane 2: Blank, 15/1 ratio nanoplexes, Lanes 3-10: 5, 15, 30, $\mathrm{min}$ and $1,4,24,48,72 \mathrm{~h}$ ). b. Binary nanoplexes. Lane 1: Free siVEGF, Lane 2: Blank, 15/1 ratio nanoplexes, Lanes 3-10; 5, 15, 30, $\min$ and 1, 4, 24, 48, 72 h). c. Free siRNA: Lane 1: Free siVEGF, Lanes 2-10: 5, 10, $15,30 \mathrm{~min}$ and $1,2,4,24,48 \mathrm{~h}$. 
Stability of siRNA in Serum One of the most major barriers for siRNA delivery is the degradation of siRNA by serum nucleases. Since siRNA can be easily degraded by nucleases either in the cytosol or circulation, instability of siRNA remains one of the obstacles for the delivery of therapeutic genes in vitro and in vivo. Therefore, for a successful systemic gene delivery, it is essential that the development of the efficient carrier to protect siRNA from degradation by serum nucleases. Complexation of siRNA with protamine and chitosan could reduce the number of accessible binding sites for nucleases and therefore provide protection against degradation. $^{21)}$

Serum stability study was performed to detect whether or not nanoplexes could protect siRNA from serum nuclease attacks. ${ }^{19)}$ As shown in Fig. 2, the gel electrophoresis results indicated that naked siRNA treated with serum was completely degraded after $4 \mathrm{~h}$ of incubation with serum at $37^{\circ} \mathrm{C}$, while the chitosan/siRNA and chitosan/siRNA/protamine nanoplexes efficiently protected siRNA from serum degredation up to $72 \mathrm{~h}$. It demonstrates that chitosan and protamine could offer protection for condensed siRNA against degradation through nanoplexes.

Characterization of the Nanoplexes Size and zeta potential measurements are formulation parameters that indicate homogeneity of the nanoplexes and can be used formulation and process optimization. In the targeted drug delivery, it is great importance of the particle size for cellular uptake. In general, particles with an average diameter between 100 and $500 \mathrm{~nm}$ could be introduced into target cells by endocytosis and subsequently permeate the nuclear membranes through the nuclear pores. ${ }^{7)}$ The physicochemical properties of positive surface charge and nanoscale particle form, facilitates electrostatic cellular membrane interaction and uptake and nuclease degradation protection. ${ }^{18)}$

In this study, size and zeta potential of nanoplexes were measured using a zetasizer The particle sizes of nanoplexes were changed between 119-462 $\mathrm{nm}$ and had a tendency to increase as the N/P ratio increased (Table 1). The particle sizes of chitosan/siRNA and protamine/siRNA nanoplexes (N/P ratio: $50 / 1$ ) were $399 \pm 13 \mathrm{~nm}$ and $462 \pm 24 \mathrm{~nm}$, respectively. In contrast, the chitosan/siRNA/protamine nanoplexes (50/1) have $282 \pm 11 \mathrm{~nm}$. The sizes of ternary nanoplexes were smaller than that of binary nanoplexes. Chitosan/siRNA/protamine nanoplexes have formed stronger compact complexes by decrease particle size. Modification of particle size depends on the balance between the binding ability of the peptide to precondense siRNA with highly cationic chitosan which would cause reduction in size. ${ }^{22)}$ In addition, complexation is accomplished by strong electrostatic interaction of the guanidinium cations with the phosphate anions of the siRNA, and one protamine fragment was reported to bind about six siRNA molecules. ${ }^{23)}$

Charge $(\mathrm{N} / \mathrm{P})$ ratio is one of factors determine particle size and zeta potential values of nanoplexes. The N/P charge ratio influence the total charge associated with the nitrogen groups of chitosan and the overall charge of the chitosan/siRNA complex and ultimately the transfection efficiency. ${ }^{24)}$ While the size of protamine/siRNA and chitosan/siRNA nanoplexes increased with the increasing chitosan and protamine amounts or N/P ratios, the size of chitosan/siRNA/protamine nanoplexes was less than the chitosan and protamine nanoplexes (Table 1). With increasing $\mathrm{N} / \mathrm{P}$ ratio, more dense nanoplexes were formed due to the cooperative effect and stronger ionic interactions. This finding in agreement with the result obtained with reported elsewhere. ${ }^{16)}$

The zeta potential of these nanoplexes increased dependently on the N/P ratio. At an $1 \mathrm{~N} / \mathrm{P}$ ratio of protamine/siRNA nanoplexes, the surface charge of nanoplex was $-17.95 \mathrm{mV}$, indicating that the arginine residue of protamine can be neutralized by the interaction with negative charges in the siRNA.

Table 1. Physical Characterization of Obtained Nanoplexes with Different Ratios

\begin{tabular}{|c|c|c|c|}
\hline $\begin{array}{l}\text { Nanoplex } \\
\text { formulation }\end{array}$ & $\mathrm{N} / \mathrm{P}$ ratio & $\begin{array}{l}\text { Zeta potential } \\
(\mathrm{mV})\end{array}$ & $\begin{array}{c}\text { Nanoplex size } \\
\text { (nm) }\end{array}$ \\
\hline Naked siVEGF & & $-23.3 \pm 1.2$ & \\
\hline Protamine & & $6.63 \pm 0.4$ & \\
\hline Chitosan & & $36.9 \pm 2.7$ & \\
\hline \multirow[t]{5}{*}{ Chitosan/siVEGF } & $1 / 1$ & $1.28 \pm 0.3$ & $135.6 \pm 2.7$ \\
\hline & $5 / 1$ & $21.1 \pm 0.5$ & $216 \pm 13.2$ \\
\hline & $10 / 1$ & $27.0 \pm 1.8$ & $232 \pm 22.2$ \\
\hline & $20 / 1$ & $52.17 \pm 2$ & $271.3 \pm 12.1$ \\
\hline & $50 / 1$ & $60.8 \pm 4.5$ & $399.5 \pm 13.4$ \\
\hline \multirow[t]{5}{*}{ Protamine/siVEGF } & $1 / 1$ & $-17.9 \pm 0.8$ & $119.1 \pm 1.9$ \\
\hline & $5 / 1$ & $2.5 \pm 1.2$ & $242.1 \pm 12.5$ \\
\hline & $10 / 1$ & $4.5 \pm 3.2$ & $259.8 \pm 13.7$ \\
\hline & $20 / 1$ & $10.6 \pm 4.5$ & $306.9 \pm 10.6$ \\
\hline & $50 / 1$ & $17.3 \pm 1.7$ & $462 \pm 18.2$ \\
\hline \multirow{5}{*}{$\begin{array}{l}\text { Chitosan/siVEGF/ } \\
\text { protamine }\end{array}$} & $1 / 1 / 1$ & $-3.7 \pm 0.5$ & $151.1 \pm 2.3$ \\
\hline & $5 / 1 / 5$ & $2.7 \pm 0.6$ & $209.3 \pm 15.2$ \\
\hline & $10 / 1 / 10$ & $5.2 \pm 0.5$ & $221.5 \pm 12$ \\
\hline & $20 / 1 / 20$ & $13.1 \pm 3.4$ & $228.1 \pm 16$ \\
\hline & $50 / 1 / 50$ & $20.8 \pm 2.4$ & $282.2 \pm 11.8$ \\
\hline
\end{tabular}

The results are expressed as the mean \pm S.D. $(n=5)$.
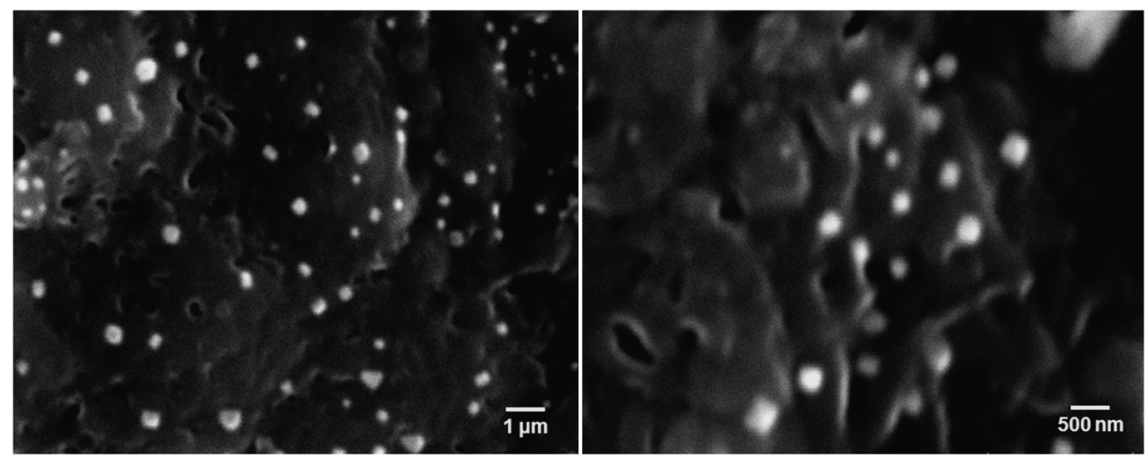

Fig. 3. SEM Photographs of the Ternary Nanoplexes 
In contrast, the surface charges of nanoplexes by increasing of $\mathrm{N} / \mathrm{P}$ ratios were changed positively (Table 1). These findings are in good agreement with those observed by other investigators. $^{25)}$ The ternary nanoplexes formed by electrostatic interactions among the positive arginine-rich domain of protamine, the positive amine groups of chitosan and the negative charge siRNA.

Morphological characterization of nanoplexes helps in understanding the relative structure and size. Ternary nanoplexes were observed under a scanning electron microscopy. Nanoplexes in round shaped were obtained and about $200 \mathrm{~nm}$ in diameters (Fig. 3).

In Vitro Cellular Uptake Successful gene silencing with RNAi depends on effective cellular delivery and cytoplasmic entry of siRNA. Moreover, the siRNA delivery systems have to be stable in the presence of serum. ${ }^{26)}$ Previous studies have shown that chitosan and protamine complexes or nanoparticles enter the cell efficiently and rapidly through a combination of specific and non-specific endocytic mechanisms. ${ }^{1,8,16-18)}$

To investigate the cellular delivery efficiency of chitosan/ siVEGF/protamine nanoplexes, we performed the cellular uptake study using FITC-labeled siVEGF. The intracellular lo-

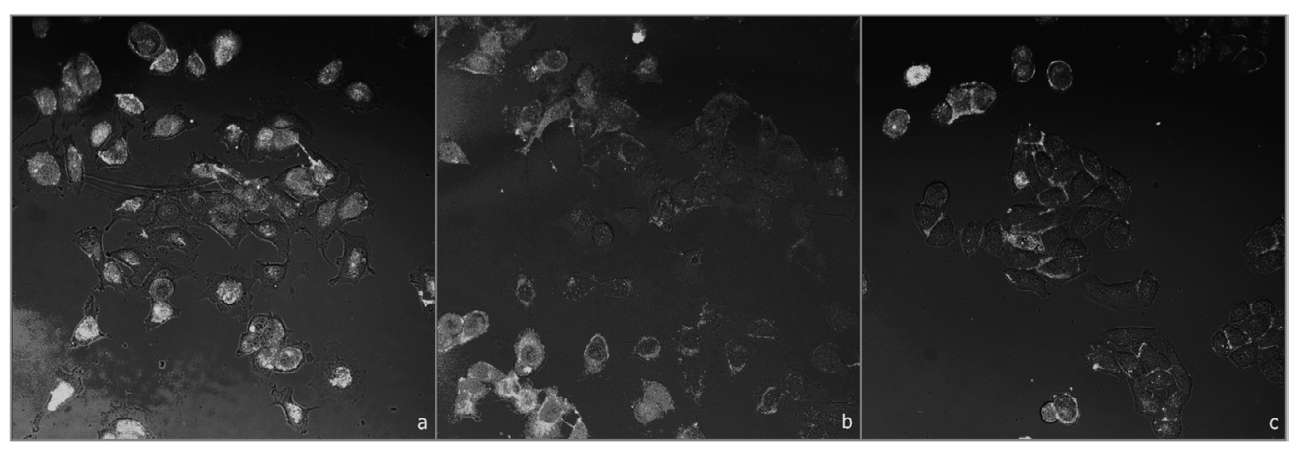

Fig. 4. Cellular Localization of Ternary and Binary Nanoplexes in MCF-7 Cells by Confocal Laser Scanning Microscopy a. Chitosan/FITC-siVEGF/protamine, b. Protamine/FITC-siVEGF and c. Chitosan/FITC-siVEGF nanoplexes (20/1).

a

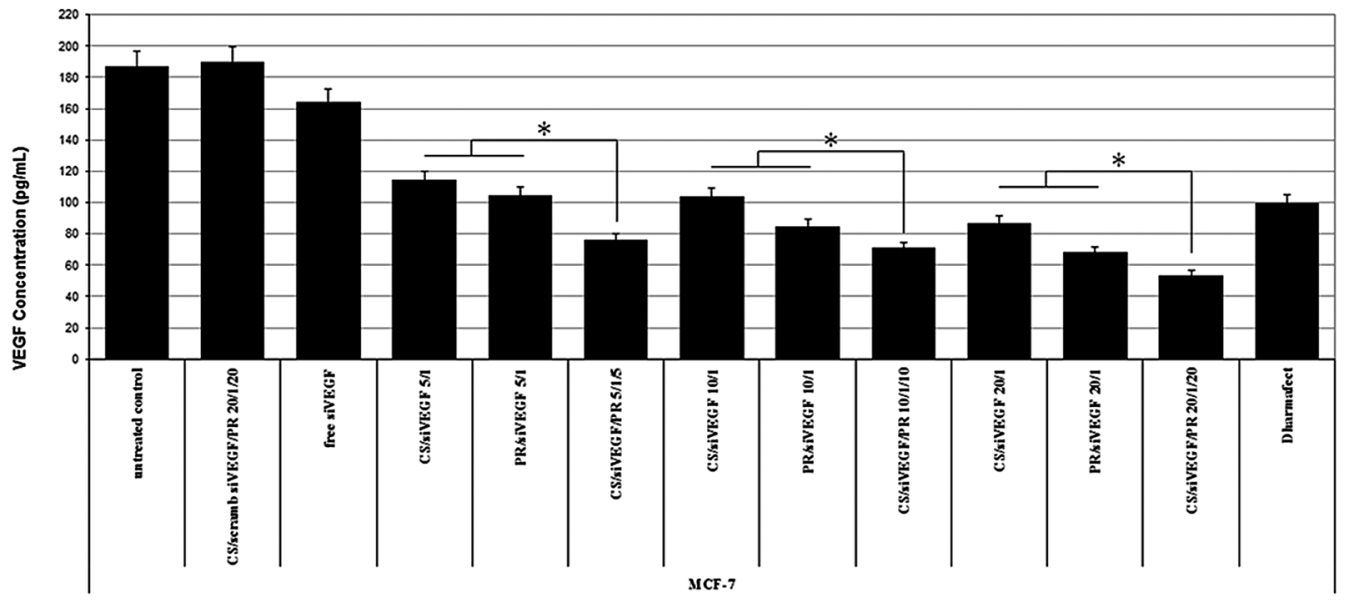

b

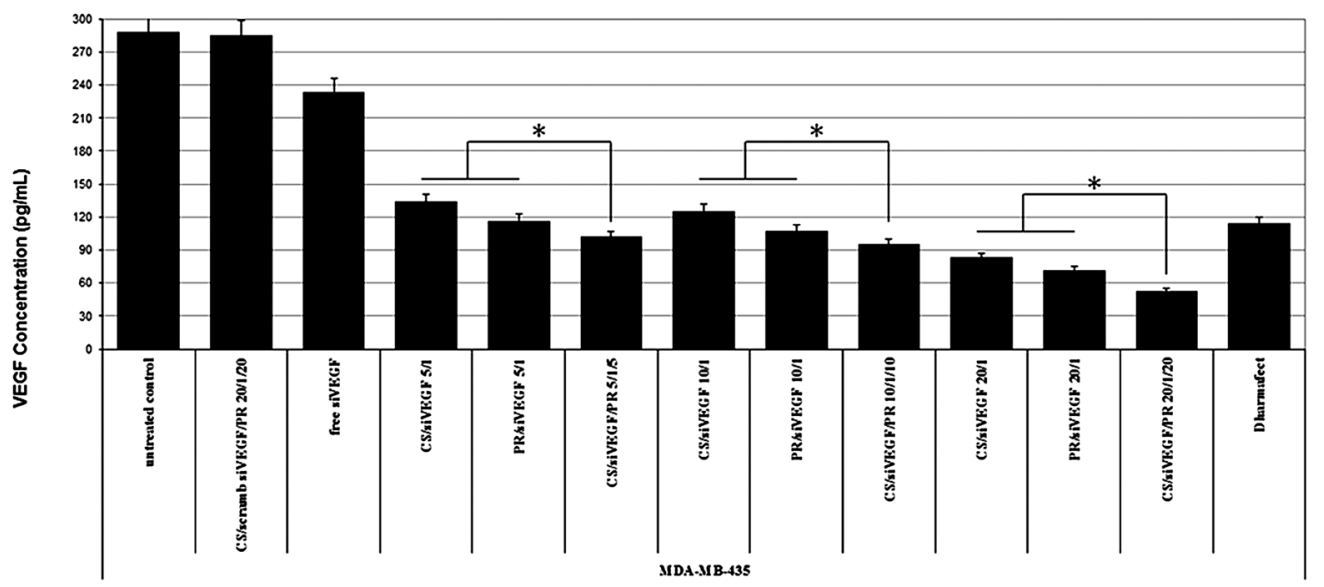

Fig. 5. Inhibition of VEGF Production with Nanoplexes at the Different Ratios and Breast Cancer Cell Lines (a. MCF-7, b. MDA-MB435 Cells)

Human VEGF concentration in the conditioned media was determined by ELISA for human VEGF. (As examined gene silencing activity in cells, silencing efficiency was estimated according to its control group for each cell.) Differences between binary and ternary nanoplexes were marked with an asterisk $(* p<0.05)$. 

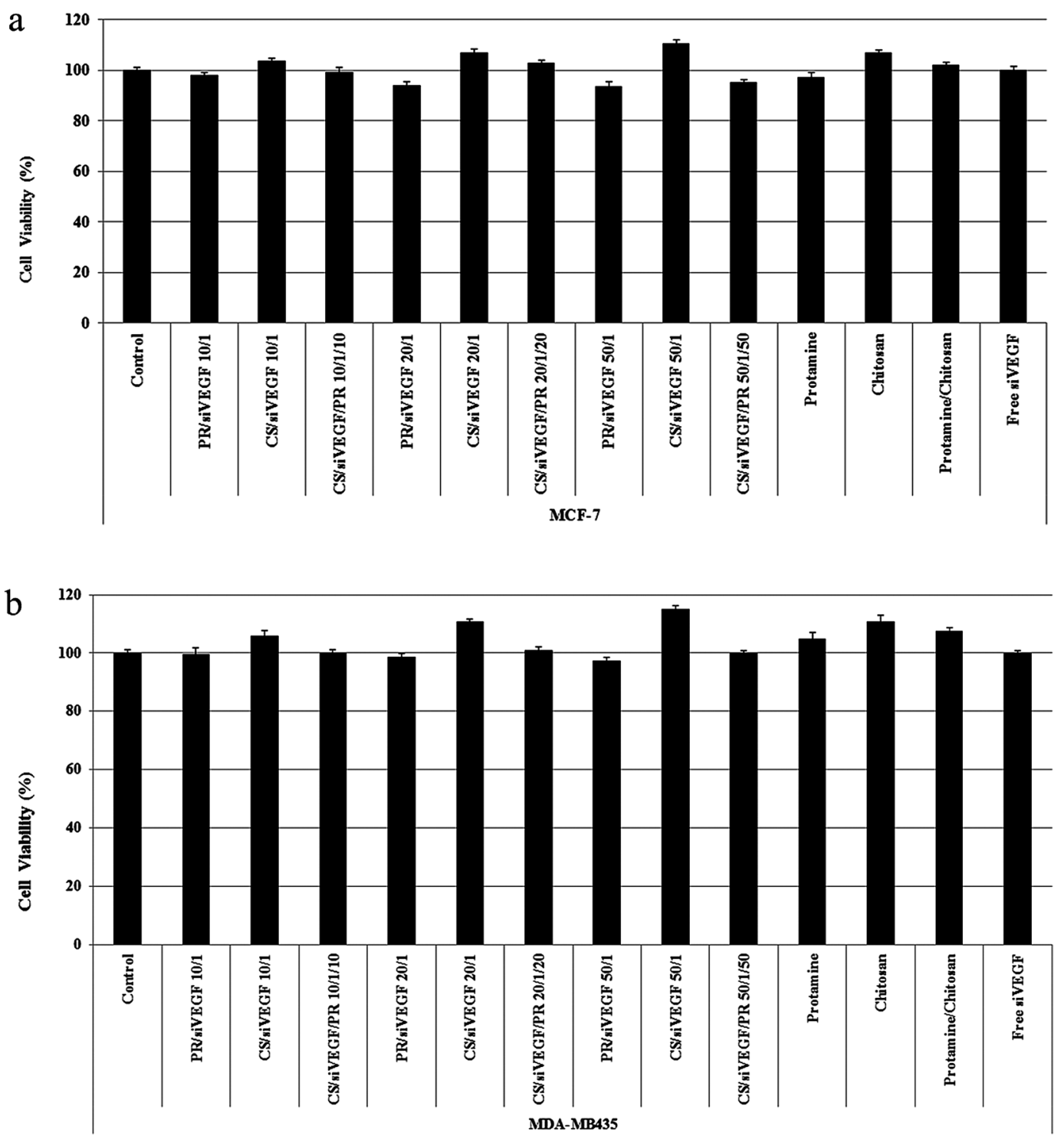

Fig. 6. a. Viability of MCF-7 and b. MDA-MB-435 Cells after Incubating with Free Chitosan $(2.5 \mathrm{mg} / \mathrm{mL})$, Free Protamine (2.5 mg/mL) and Binary and Ternary Nanoplexes at Different N/P Ratios

After treatment of the cells upon $48 \mathrm{~h}$, cell viability was measured by MTT assay. The results are expressed as the mean \pm standard deviation (S.D.) of four independent experiments.

calization of the ternary and binary nanoplexes in MCF-7 cells was confirmed by confocal microscopy after $4 \mathrm{~h}$ incubation of the nanoplexes with the cells (Fig. 4). Figure 4a shows that ternary nanoplexes internalized into the cell as efficiently. Ternary nanoplexes were seen to translocate through cell membranes and accumulate in the cytoplasm and nucleus. These findings suggest that siRNA require specific formulations to cross cellular membranes. Ternary nanoplexes promotes uptake of siRNA to shield the negatively charge backbone of siRNA by highly cationic properties.

In Vitro VEGF Gene Silencing and Cytotoxicity The in vitro transfection efficiency of the binary and ternary nanoplexes was evaluated by sequence-specific VEGF gene silencing activity in breast cancer cell lines. The effects of different nanoplex formulations containing siVEGF on VEGF secretion from cells were investigated using ELISA.

As shown in Fig. 5, while the nanoplex formulations showed moderate to strong gene silencing effect, naked siRNA showed minimal effect $(p<0,05)$. Because, the siVEGF was not by itself knockdown the VEGF expression, indicating the cell membrane translocation by chitosan or protamine was critical for the effective VEGF silencing by the siVEGF.

The depending on the N/P ratio of the gene silencing efficiency was seen in Fig. 5, increasing the N/P ratio of nanoplexes enhanced the suppression of VEGF secretion in cells. Moreover, ternary nanoplexes exhibit higher than silencing efficiency than binary nanoplexes $(p<0.05)$. While in vitro gene silencing effect was observed $71 \%$ and $74 \%$ after transfection of chitosan/siVEGF (20/1) and protamine/siVEGF (20/1), it was obtained $83 \%$ after transfection of chitosan/siVEGF/protamine (20/1/20) nanoplexes in MDA-MB435 cell line (Fig. 5). Furthermore, ternary nanoplexes exhibited greater inhibition on VEGF secretion than Dharmafect. The incorporation of protamine to the chitosan is promoted not only to condensation and transfection of siRNA but also to nuclear localization and stabilization against enzyme degredation. In addition, protamine is used as transfection accelerator in the gene delivery. ${ }^{27,28)}$ Therefore, ternary nanoplexes containing protamine 
and chitosan possess important advantages as an efficient carrier for delivering therapeutics.

Scrambled siRNA nanoplexes did not show any gene inhibition comparable to siVEGF nanoplexes, suggesting that siVEGF can suppress VEGF expression in cancer cells in a highly sequence-specific manner. In vitro silencing effect of ternary nanoplexes in the cells as follows MDA-MB-435 (83\%) $>$ MCF-7 (71\%) (Fig. 5). Ternary nanoplexes mediated suppression of VEGF secretion from the tumor cells was striking when compared with the untreated.

The results of in vitro ELISA were consistent with the cellular uptake assays which showed that the chitosan/siRNA/ protamine nanoplexes with higher cellular uptake exhibited higher levels of gene silencing. The gene transfection efficiency of ternary nanoplexes was enhanced by incorporated protamine to the chitosan. Protamine is a cationic small protein that act as a nuclear localization signal and has facilitate the intracellular release of siRNA and DNA. It is promoted the release of genetic material to the cytoplasm and the penetration of DNA into the nucleus. ${ }^{22)}$ Confocal analysis showed that cellular uptake of ternary nanoplexes in the cells was higher than binary nanoplexes.

Our results are in accordance with earlier reports. Choi et al. reported that VEGF protein level was significantly reduced when the cells were treated with low MW protamine/siRNA complex and the inhibitory effect of these complexes was dose dependent. ${ }^{16)}$

To evaluate the safety of protamine and chitosan nanoplexes, MTT assay was used to determine cell viability after transfection of the nanoplexes at the different $\mathrm{N} / \mathrm{P}$ ratios in MCF-7 and MDA-MB-435 cell lines (Fig. 6). In addition, protamine and chitosan alone and both were studied to monitor whether or not non-specific effect on VEGF levels as a consequence of cytotoxicity. The results of the MTT assay suggested that binary and ternary nanoplexes at any N/P ratio did not show cytotoxicity. Moreover, chitosan and protamine alone and both did not affect cell viability and did not inhibit VEGF level in cells as reported by Choi et al. ${ }^{16)}$ This result indicated that chitosan/siRNA/protamine nanoplexes are a highly efficient and safe carrier for siRNA.

\section{CONCLUSION}

Our results indicated that chitosan/siRNA/protamine nanoplexes could promote the delivery of siRNA into the tumor cells and may be useful for clinical delivery of therapeutic siRNAs. The data in this study demonstrate that ternary nanoplexes are increased not only cellular uptake of siRNA, but also inhibition of VEGF expression. Ternary nanoplexes have led to the formation of a better delivery system for siRNA with higher transfection efficiency compared to binary nanoplexes.

Acknowledgment This study was supported by Marmara University Scientific Research Projects Association (SAG-D-140312-0043).

\section{REFERENCES}

1) Guo J, Bourre L, Soden DM, O'Sullivan GC, O'Driscoll C. Can non-viral technologies knockdown the barriers to siRNA delivery and achieve the next generation of cancer therapeutics? Biotechnol. Adv., 29, 402-417 (2011).

2) Abdelrahim M, Safe $S$, Baker $C$, Abudayyeh A. RNAi and cancer: Implications and applications. J. RNAi Gene Silencing, 2, 136-145 (2006).

3) Oh YK, Park TG. siRNA delivery systems for cancer treatment. Adv. Drug Deliv. Rev., 61, 850-862 (2009).

4) Masiero $M$, Nardo $G$, Indraccolo $S$, Favaro E. RNA interference: Implications for cancer treatment. Mol. Aspects Med., 28, 143-166 (2007).

5) David S, Pitard B, Benoît JP, Passirani C. Non-viral nanosystems for systemic siRNA delivery. Pharmacol. Res., 62, 100-114 (2010).

6) de Martimprey H, Vauthier C, Malvy C, Couvreur P. Polymer nanocarriers for the delivery of small fragments of nucleic acids: Oligonucleotides and siRNA. Eur. J. Pharm. Biopharm., 71, 490-504 (2009).

7) Reischl D, Zimmer A. Drug delivery of siRNA therapeutics: potentials and limits of nanosystems. Nanomedicine, 5, 8-20 (2009).

8) Mao S, Sun W, Kissel T. Chitosan-based formulations for delivery of DNA and siRNA. Adv. Drug Deliv. Rev., 62, 12-27 (2010).

9) Pillé JY, Li H, Blot E, Bertrand JR, Pritchard LL, Opolon P, Maksimenko A, Lu H, Vannier JP, Soria J, Malvy C, Soria C. Intravenous delivery of anti-RhoA smal interfering RNA loaded in nanoparticles of chitosan in mice: safety and efficacy in xenografted aggressive breast cancer. Hum. Gene Ther., 17, 1019-1026 (2006).

10) Şalva E, Kabasakal L, Eren F, Özkan N, Çakalağaoğlu F, Akbuğa J. Local delivery of chitosan/VEGF siRNA nanoplexes reduces angiogenesis and growth of breast cancer in vivo. Nuc. Acid Ther., 22, 40-48 (2012).

11) Howard KA, Rahbek UL, Liu X, Damgaard CK, Glud SZ, Andersen MO, Hovgaard MB, Schmitz A, Nyengaard JR, Besenbacher F, Kjems J. RNA interference in vitro and in vivo using a novel chitosan/siRNA nanoparticle system. Mol. Ther., 14, 476-484 (2006).

12) Jere D, Jiang HL, Kim YK, Arote R, Choi YJ, Yun C, Cho MH, Cho CS. Chitosan-graft-polyethylenimine for Akt1 siRNA delivery to lung cancer cells. Int. J. Pharm., 378, 194-200 (2009).

13) Chen J, Yu Z, Chen H, Gao J, Liang W. Transfection efficiency and intracellular fate of polycation liposomes combined with protamine. Biomaterials, 32, 1412-1418 (2011).

14) DeLong RK, Akhtar U, Sallee M, Parker B, Barber S, Zhang J, Craig M, Garrad R, Hickey AJ, Engstrom E. Characterization and performance of nucleic acid nanoparticles combined with protamine and gold. Biomaterials, 30, 6451-6459 (2009).

15) Song E, Zhu P, Lee SK, Chowdhury D, Kussman S, Dykxhoorn DM, Feng Y, Palliser D, Weiner DB, Shankar P, Marasco WA, Lieberman J. Antibody mediated in vivo delivery of small interfering RNAs via cell-surface receptors. Nat. Biotechnol., 23, 709-717 (2005).

16) Choi YS, Lee JY, Suh JS, Kwon YM, Lee SJ, Chung JK, Lee DS, Yang VC, Chung CP, Park YJ. The systemic delivery of siRNAs by a cell penetrating peptide, low molecular weight protamine. Biomaterials, 31, 1429-1443 (2010).

17) Wang T, Upponi JR, Torchilin VP. Design of multifunctional nonviral gene vectors to overcome physiological barriers: Dilemmas and strategies. Int. J. Pharm., 427, 3-20 (2012).

18) Howard KA. Delivery of RNA interference therapeutics using polycation-based nanoparticles. Adv. Drug Deliv. Rev., 61, 710-720 (2009).

19) Park YJ, Liang JF, Ko KS, Kim SW, Yang VC. Low molecular weight protamine as an efficient and nontoxic gene carrier: in vitro study. J. Gene Med., 5, 700-711 (2003).

20) Yuan H, Zhang W, Du YZ, Hu FQ. Ternary nanoparticles of anionic lipid nanoparticles/protamine/DNA for gene delivery. Int. J. Pharm., 392, 224-231 (2010).

21) Dinauer N, Lochmann D, Demirhan I, Bouazzaoui A, Zimmer A, Chandra A, Kreuter J, Briesen H. Intracellular tracking of prot- 
amine/antisense oligonucleotide nanoparticles and their inhibitory effect on HIV-1 transactivation. J. Control. Release, 96, 497-507 (2004).

22) Delgado D, Pozo-Rodríguez A, Solinís MA, Rodríguez-Gascón A. Understanding the mechanism of protamine in solid lipid nanoparticle-based lipofection: The importance of the entry pathway. Eur. J. Pharm. Biopharm., 79, 495-502 (2011).

23) Mayer G, Vogel V, Weyermann J, Lochmann D, van den Broek JA, Tziatzios C, Haase W, Wouters D, Schubert US, Zimmer A, Kreuter J, Schubert D. Oligonucleotide-protamine albumin nanoparticles: protamine sulfate causes drastic size reduction. J. Control. Release, 106, 181-187 (2005).

24) Rudzinski WE, Aminabhavi TM. Chitosan as a carrier for targeted delivery of small interfering RNA. Int. J. Pharm., 399, 1-11 (2010).
25) Tanaka K, Kanazawa T, Ogawa T, Takashima Y, Fukuda T, Okada H. Disulfide crosslinked stearoyl carrier peptides containing arginine and histidine enhance siRNA uptake and gene silencing. Int. J. Pharm., 398, 219-224 (2010).

26) Patil Y, Panyam J. Polymeric nanoparticles for siRNA delivery and gene silencing. Int. J. Pharm., 367, 195-203 (2009).

27) Vighi E, Montanari M, Ruozi B, Tosi G, Magli A, Leo E. Nuclear localization of cationic solid lipid nanoparticles containing protamine as transfection promoter. Eur. J. Pharm. Biopharm., 76, 384-393 (2010).

28) Tsuchiya Y, Ishii T, Okahata Y, Sato T. Characterization of protamine as a transfection accelerator for gene delivery. J. Bioact. Compat. Polym., 21, 519-537 (2006). 